\title{
All-Trans Retinoic Acid Inhibits Migration and Invasiveness of Rheumatoid Fibroblast-Like Synoviocytes
}

\author{
Nerea Mosquera, Angela Rodriguez-Trillo, Francisco J. Blanco, Antonio Mera-Varela, \\ Antonio Gonzalez, and Carmen Conde
}

Laboratorio de Reumatología Experimental y Observacional, y Servicio de Reumatología, Instituto de Investigación Sanitaria de Santiago (IDIS), Hospital Clínico Universitario de Santiago de Compostela (CHUS), Servizo Galego de Saude (SERGAS), Travesía da Choupana s/n, Santiago de Compostela, Spain (N.M., A.R.-T., A.G., C.C.); Servicio de Reumatología, Instituto de Investigacion Biomedica de A Coruña (INIBIC)-Hospital Universitario A Coruña, A Xubias 84, A Coruña, Spain (F.J.B.); and Servicio de Reumatología, Instituto de Investigación Sanitaria de Santiago (IDIS), Hospital Clínico Universitario de Santiago de Compostela (CHUS), Servizo Galego de Saude (SERGAS), Travesía da Choupana s/n, Santiago de Compostela, Spain (A.M.-V.)

Received July 9, 2019; accepted November 26, 2019

\begin{abstract}
Fibroblast-like synoviocytes (FLSs) are pivotal in inflammation and joint damage of rheumatoid arthritis (RA). They acquire an active and aggressive phenotype, displaying increased migration and invasiveness and contributing to perpetuate synovial inflammation and destruction of cartilage and bone. The main current therapies of RA are focused against inflammatory factors and immune cells; however, a significant percentage of patients do not successfully respond. Combined treatments with drugs that control inflammation and that reverse the pathogenic phenotype of FLS could improve the prognosis of these patients. An unexplored area includes the retinoic acid, the main biologic retinoid, which is a candidate drug for many diseases but has reached clinical use only for a few. Here, we explored the effect of all-trans retinoic acid (ATRA) on the aggressive phenotype of FLS from patients with RA. RA FLSs were treated with ATRA, tumor necrosis factor (TNF), or TNF+ATRA, and cell migration and invasion were analyzed. In addition, a microarray analysis of expression, followed by gene-set analysis and quantitative
\end{abstract}

polymerase chain reaction validation, was performed. We showed that ATRA induced a notable decrease in FLS migration and invasion that was accompanied by complex changes in gene expression. At supraphysiological doses, many of these effects were overridden or reverted by the concomitant presence of TNF. In conclusion, these results have demonstrated the therapeutic potential of retinoic acid on RA FLS provided TNF could be counterbalanced, either with high ATRA doses or with TNF inhibitors.

\section{SIGNIFICANCE STATEMENT}

All-trans retinoic acid (ATRA) reduced the rheumatoid arthritis (RA) fibroblast-like synoviocyte migration and invasiveness and down-regulated gene expression of cell motility and migration genes. At supraphysiological doses, some of these effects were reverted by tumor necrosis factor. Therefore, ATRA could be an RA drug candidate that would require high doses or combined treatment with anti-inflammatory drugs.

\section{Introduction}

Rheumatoid arthritis (RA) is an autoimmune disease characterized by inflammation of the peripheral joints involving synovitis and cartilage and bone degradation (Klareskog et al., 2009; McInnes and Schett, 2011). The resident synovial cells, macrophages and fibroblast-like synoviocytes (FLSs), contribute to the disease pathogenesis in multiple ways. Specifically, FLSs acquire an activated status, leading to secretion of

This work was supported by Fondo de Investigación Sanitaria, Instituto de Salud Carlos III, with participation of European Regional Development Fund (FEDER) funds (European Union) [Grants PI1701660 and PI1401153 and by Redes Tematicas de Investigacion Cooperativa en Salud (RETICS) Program, RD16/0012/0014].

https://doi.org/10.1124/jpet.119.261370. proinflammatory mediators, cytokines, and chemokines together with metalloproteases, cathepsins, and other factors contributing to cartilage and bone erosion. In addition, RA FLS acquires some features of transformed cells that are described as unregulated proliferation, resistance to apoptosis, and insensitivity to contact inhibition. These characteristics contribute to cartilage and bone damage by the invasion of the hyperplasic and aggressive synovia. This is a stable phenotype maintained by epigenetic marks and somatic mutations that seems critical for disease persistence and that differentiates RA FLS from healthy FLS (Bartok and Firestein, 2010; Neumann et al., 2010; Ospelt, 2017). Preclinical models have shown the effectiveness of targeting RA FLS, though none of the tested drugs have yet reached clinical use. In contrast, available drugs target the immune and inflammatory

ABBREVIATIONS: ATRA, all-trans retinoic acid; COL, collagen; CXCL3, C-X-C motif chemokine ligand 3; CXCL9, C-X-C motif chemokine ligand 9; CX3CL1, C-X3-C motif chemokine ligand 1; EPSTI1, epithelial stromal interaction 1; FLS, fibroblast-like synoviocyte; GSEA, Gene Set Enrichment Analysis; IL, interleukin; MMP1, matrix metalloprotease 1; NES, normalized enrichment score; NID2, nidogen 2; NOD2, nucleotide-binding oligomerization domain 2; PCR, polymerase chain reaction; PTX3, pentraxin 3; RA, rheumatoid arthritis; ST3GAL5, ST3 beta-galactoside alpha-2, 3sialyltransferase 5; TBP, TATA-box-binding protein; TNF, tumor necrosis factor; VCAM1, vascular cell adhesion protein 1. 
components of RA, but as monotherapy often they are insufficient to achieve remission or adequate control of the disease activity (Smolen et al., 2017). This circumstance has motivated the common use of drug combinations, with the most frequent including methotrexate together with a targeted biologic drug as one of the monoclonal anti-tumor necrosis factor (TNF) antibodies (Smolen et al., 2017). A sizable fraction of patients does not reach adequate response even with these drug combinations. Therefore, there is a growing interest in developing treatments aimed to revert the activated phenotype of RA FLS (Niedermeier et al., 2010). Recently, we found that a transporter of retinoids, cellular retinoic acid binding protein 2 , is a candidate drug target of this type because cellular retinoic acid binding protein 2 suppression reverts RA FLS resistance to apoptosis (Mosquera et al., 2018). In the same study, we observed unconventional responses of RA FLS to the main biologic retinoid, all-trans retinoic acid. These results prompted us to investigate further the effects of alltrans retinoic acid (ATRA) on the activated phenotype of RA FLS (Mosquera et al., 2018).

Vitamin A and retinoids are generic designations for an array of essential organic molecules that include retinal, retinol, and retinoic acid. They are needed for the visual system, cell homeostasis and differentiation, integrity of epithelia, erythropoiesis, reproduction, lymph node morphogenesis, response to infection, and other immune functions (Al Tanoury et al., 2013; Coyle et al., 2013). Some retinoids have been used since long ago to treat psoriasis, in which they act on keratinocyte differentiation and proliferation and on the balance between different cluster of differentiation 4 subsets (Brown and Noelle, 2015). In addition, retinoids have been considered for the treatment of several types of cancer and autoimmune diseases. In cancer cells, retinoids induce differentiation, cell cycle arrest, apoptosis, and inhibition of cell migration and invasiveness (Flamini et al., 2014; Young et al., 2015; Cui et al., 2016; Li et al., 2017). In autoimmune diseases, retinoids have shown improvement of animal models of multiple sclerosis (Massacesi et al., 1991), systemic lupus erythematosus (Perez de Lema et al., 2004), type 1 diabetes (Wang et al., 2016), and RA (Kuwabara et al., 1996; Kwok et al., 2012). However, progress to further development of retinoids as new drugs for these diseases has been slow. In the case of RA animal models, some studies have shown disease aggravation in place of improvement (Trentham and Brinckerhoff, 1982, Beehler et al., 2003). The current study was started to explore the effects of ATRA on the key features of the RA FLS phenotype, namely cell migration and invasion. Given that TNF is one of the most relevant cytokines in RA pathogenesis, as supported by the efficacy of anti-TNF treatments, the experiments also involved analysis of the potential influence of TNF on the results.

The principal result of this work has been the discovery of selective modulation by ATRA of several aspects of the RA FLS phenotype. This regulation produces beneficial effects, such as decreased migration and invasion. However, ATRA effects on RA FLS were in many instances overridden or reverted by the concomitant presence of TNF. The TNF overturning of ATRA effects was observed at supraphysiological doses of ATRA, but a decreased migration could be restated by further increasing the ATRA dose. This need for counterbalancing TNF modulation should inform the design of treatment trials of ATRA and further studies of its role in RA pathogenesis.

\section{Materials and Methods}

Patients and Cell Culture. FLSs were derived from synovial tissue obtained from 11 patients with RA (eight women and three men) showing acute synovitis and undergoing synovectomy by clinical indication and with independency of this study. The patients fulfilled the American College of Rheumatology criteria for the classification of RA (Arnett et al., 1988) and provided their informed written consent. The study was performed according to the recommendations of the Declaration of Helsinki and was approved by the Comité Ético de Investigación Clínica de Galicia (Approval No. 2014/393).

The synovial tissue was enzymatically digested, and the adherent cells were grown to $80 \%-90 \%$ confluence in Dulbecco's modified Eagle's medium with $10 \%$ FBS, $1 \%$ glutamine, and $1 \%$ penicillin/ streptomycin, as previously described (Orosa et al., 2012). Once confluent, cultures were trypsinized and diluted at a 1:3 split ratio for a new passage. The experiments were restricted to FLSs from passages 3 to 8 because FLSs become a homogeneous population at passage 3 and keep their phenotype to passages 8 to 9 (Lories et al., 2003; Rosengren et al., 2007).

Migration Assay. The capacity of RA FLS to migrate was analyzed with a cell wound closure assay. We used the Ibidi Culture Inserts (Ibidi, Martinsried, Germany) to improve the reproducibility of the results. These inserts, composed of two chambers separated by a $500 \mu \mathrm{m}$-thick wall, were placed into each of the 24 wells of the plate. FLSs were seeded into the chambers of the inserts $\left(5 \times 10^{3}\right.$ cells/chamber $)$ and cultured until confluence was reached. At this time, the inserts were removed to create the cell-free gap (time $=0$ hour). Also at this moment, the culture medium was replaced by a serum-deprived (1\% FBS) medium containing ATRA $(5 \mu \mathrm{M})$, TNF (10 ng/ml), ATRA+TNF (5 $\mu \mathrm{M}$ and $10 \mathrm{ng} / \mathrm{ml}$, respectively), or DMSO (0.05\%) and cells were incubated for 96 hours. Photographs were taken under a microscope Axio Vert.A1 (Zeiss, Oberkochen, Alemania) at 0 and 96 hours, and the fraction of the initial gap that was filled with cells at 96 hours was quantified with the Image J software (National Institutes of Health).

Transwell Invasion Assay. The ability of RA FLS to invade extracellular matrix was tested using the 24-Well Milicell Hanging Cell Culture Inserts (Merck Millipore, Darmstadt, Germany) covered with Matrigel (BD Biosciences, Franklin Lakes, NJ). The inserts create a Boyden chamber separated by a membrane of polyethylene terephthalate with $8.0-\mu \mathrm{m}$ pore size. This membrane was coated with $200 \mu \mathrm{g} / \mathrm{ml}$ of Matrigel, which mimics the extracellular matrix. The FLSs $\left(5 \times 10^{4}\right.$ cells in $\left.200 \mu \mathrm{l}\right)$ were suspended in a serum-deprived medium (1\% FBS) containing ATRA $(5 \mu \mathrm{M})$, TNF (10 ng/ml), ATRA+TNF ( $5 \mu \mathrm{M}$ and $10 \mathrm{ng} / \mathrm{ml}$, respectively), or DMSO $(0.05 \%)$ and plated in the upper chamber. Simultaneously, culture medium containing $10 \%$ FBS $(500 \mu \mathrm{l})$ was placed in the lower chamber as a chemoattractant. Afterward, the system was incubated for 48 hours. At this time, the cells were fixed with paraformaldehyde, stained with Giemsa, and photographed under the microscope. The number of cells in the lower side of the membrane in 10 random fields at $200 \times$ magnification was counted with the help of the ImageJ software.

Microarray Analysis. Gene expression analysis was performed with the SurePrint G3 $8 \times 60 \mathrm{~K}$ v3 ID: 072363 (Agilent Technologies, CA) one-color microarray following the manufacturer protocols. This analysis involved the four culture conditions compared in previous experiments: ATRA $(5 \mu \mathrm{M}), \mathrm{TNF}(10 \mathrm{ng} / \mathrm{ml})$, ATRA+TNF $(5 \mu \mathrm{M}$ and $10 \mathrm{ng} / \mathrm{ml}$, respectively), or DMSO $(0.05 \%)$. Here, the FLSs from six patients were cultured for 12 hours with the different treatments in the serum-deprived medium. Total RNA was obtained using the Speedtools total RNA extraction Kit (Biotools, Madrid, Spain). Its integrity was assessed with the Agilent 2100 Bioanalyzer in combination with Agilent RNA 6000 Nano Chips. Subsequently, RNA was pooled (two pools of three patients each), and $1 \mu \mathrm{g}$ of total RNA was subjected to cDNA synthesis and cyanine 3-CTP labeling using Agilent's Low Input Quick-In-One Kit. The product was hybridized on the microarray using SureHyb hybridization chambers (Agilent). Once the microarray was processed, fluorescence data 
were extracted with the Agilent Feature Extraction software and normalized with the GeneSpring GX 13.0 software (Agilent). However, no comparisons of expression data were done at the individual gene level. In its place, a gene set functional analysis was performed. It was based in the rank product order of gene expression, which was provided as the preranked input for the Gene Set Enrichment Analysis (GSEA) algorithm. We used the Hallmark gene sets of the Molecular Signatures Database, which summarize and represent specific well-defined biologic states or processes and display coherent expression. The names of the gene sets do not fully represent their content, which is available at http://software.broadinstitute. org/gsea/msigdb/genesets.jsp?collection $=$ H. A gene set was considered significantly enriched if its normalized enrichment score (NES) $\geq 3$ [all of them showed family-wise error rate $\left(<10^{-4}\right)$ ]. GSEA and Molecular Signatures Database have been developed and are maintained at the Broad Institute of the Massachusetts Institute of Technology (http://www.broad.mit.edu/gsea/).

Real-Time Quantitative Polymerase Chain Reaction. Realtime quantitative polymerase chain reaction (PCR) was performed in a Rotor-Gene (Corbett Research, Australia), using 1-Step QRTPCRBrilliant III SYBR Green (Agilent Technologies), according to the manufacturer's protocol. Relative levels of gene expression were normalized to the TATA-box-binding protein (TBP) gene using the comparative $\mathrm{C}_{t}$ method, in which $\mathrm{C}_{\mathrm{t}}$ is the cycle at which the amplification is initially detected. The relative amount of mRNA was calculated according to the $2^{-\Delta \Delta \mathrm{Ct}}$ method, wherein: $\Delta \mathrm{C}_{\mathrm{t}}=\mathrm{C}_{\mathrm{t}}$ target $-\mathrm{C}_{\mathrm{t}}$ TBP and $\Delta \Delta \mathrm{C}_{\mathrm{t}}=\left(\mathrm{C}_{\mathrm{t} \text { target }}-\mathrm{C}_{\mathrm{t} \text { TBP }}\right)_{\text {Basal }}-\left(\mathrm{C}_{\mathrm{t} \text { target }}-\mathrm{C}_{\mathrm{t} \text { TBP }}\right)_{\text {Treatment }}$.

In this way, $\Delta \Delta \mathrm{C}_{\mathrm{t}}=0$, and $2^{\circ}=1$ for RA FLS incubated with DMSO $0.05 \%$. For the experimental treatments, the value $2^{-\Delta \mathrm{Ct}}$ indicates gene expression relative to TBP and the value $2^{-\Delta \Delta \mathrm{Ct}}$ indicates the fold change in gene expression relative to the control. The primers were obtained for the database PrimerBank (Table 1) and manufactured by Sigma-Aldrich.

Data Availability. Data from microarray study have been deposited in National Center for Biotechnology Information's Gene Expression Omnibus and are accessible through the GEO Series accession number [GEO: GSE120785]. To review go to: https://www. ncbi.nlm.nih.gov/geo/query/acc.cgi?acc $=$ GSE120785. Enter token ilitwayybfqjyz into the box.

The rest of the data generated during this study is available upon request from the authors.

Statistical Analysis. Differences between experimental groups were assessed with the Mann-Whitney $U$ test or the Wilcoxon matched-pairs test. A value of $P<0.05$ was considered significant. Analyses were performed with GraphPad Prism version 5.00 for Windows (GraphPad Software, San Diego, CA).

\section{Results}

ATRA Reduces Migration and Invasiveness of RA FLS. We have analyzed whether the treatment with ATRA could reduce the migration ability of RA FLS using a cell wound-healing assay. We used the dose of $5 \mu \mathrm{M}$ ATRA based on previous literature (Chen and Stallings, 2007; Das et al., 2010, 2013; Foley et al., 2011; García-Regalado et al., 2013) and our previous work (Mosquera et al., 2018). When the FLS cells were cultured with ATRA, the healed area was reduced to about half the control at 96 hours $(P<0.01$, Fig. 1 , A and B). In contrast, the cell wound-healing assay in the presence of TNF showed increased RA FLS migration, and this increase was not overcome by the addition of ATRA in the ATRA+TNF assays (Fig. 1, A and B).

In a second step, we tested whether the reduction of migration with ATRA correlated with similar changes in FLS invasion. For these experiments, invasion through an extracellular matrix mimic, Matrigel, was used. The average result of FLS from nine patients with RA showed a significantly reduced number of invading cells (30\% fewer FLSs) with $5 \mu \mathrm{M}$ ATRA compared with the control (Fig. $2, P<0.05$ ). This decrease in invasiveness was similar to that observed in the presence of TNF and not significantly different to that found in the presence of ATRA+TNF (Fig. 2, A and B).

Identification of ATRA and ATRA+TNF Regulated Gene Sets by Microarray Analysis. In an attempt to discover clues explaining the previous results at the molecular level, a microarray study was performed. It included one-color microarrays containing about 60,000 probes that were hybridized with cDNA from FLS cultured in four conditions: control medium, ATRA, TNF, and ATRA+TNF. Two pools, including FLS from three different patients with RA each, were prepared with the RNAs and hybridized with the array. The results were used to screen for gene sets that were specifically modified by ATRA (either in the comparison of ATRA with the control or in the comparison of ATRA+TNF vs. TNF) or by the interaction between ATRA and TNF (showing differential effects with ATRA+TNF compared with that observed either with ATRA or with TNF).

Some gene sets showed evidence of interaction between ATRA and TNF effects leading to differential modulation of expression (Fig. 3). Two of them pertaining to interferon gamma response and interferon alpha response were enriched in genes up-regulated by TNF, ATRA, or ATRA+TNF relative to their paired condition except in the ATRA+TNF versus TNF comparison, wherein ATRA+TNF was associated with lower expression than TNF (Fig. 3). Another gene set showing evidence of interaction between ATRA and TNF concerned the epithelial-mesenchymal transition. The genes in this set were down-regulated by ATRA compared with the control, but up-regulated by ATRA+TNF in relation with ATRA. This latter result was observed in spite of the absence of a significant effect of TNF on its own (Fig. 3). Some of the

TABLE 1

Primer sets used for quantitative PCR study

\begin{tabular}{lll}
\hline Gene & \multicolumn{1}{c}{ Primers Forward } & Primers Reverse \\
\hline CX3CL1 & ACCACGGTGTGACGAAATG & TGTTGATAGTGGATGAGCAAAGC \\
CXCL3 & TGGTCACTGAACTGCGCT & ATGCGGGGTTGAGACAAG \\
EPSTI1 & AGCAGGAGCTGGCCAACCTGGA & TTGTGGGCCACAAACAGCACT \\
VCAM1 & GGGAAGATGGTCGTGATCCTT & TCTGGGGTGGTCTCGATTTTA \\
PTX3 & TTATTCCCAATGCGTTCCAAGA & GCACTAAAAGACTCAAGCCTCAT \\
NOD2 & CACCGTCTGGAATAAGGGTACT & TTCATACTGGCTGACGAAACC \\
MMP1 & CTCTGGAGTAATGTCACACCTCT & TGTTGGTCCACCTTTCATCTTC \\
IL1B & CGTTATCCCATGTGTCGAAGAA \\
ST3GAL5 & AGCTACGAATCTCCGACCAC & GGAGTAAGTCCACGCTATACCT \\
CXCL9 9 & AGGAATGTCGTCCCAAGTTTG & AGGGCTTGGGGCAAATTGT \\
TBP & CCAGTAGTGAGAAAGGGTCGC & CACATCACAGCTCCCCACCA \\
\hline
\end{tabular}



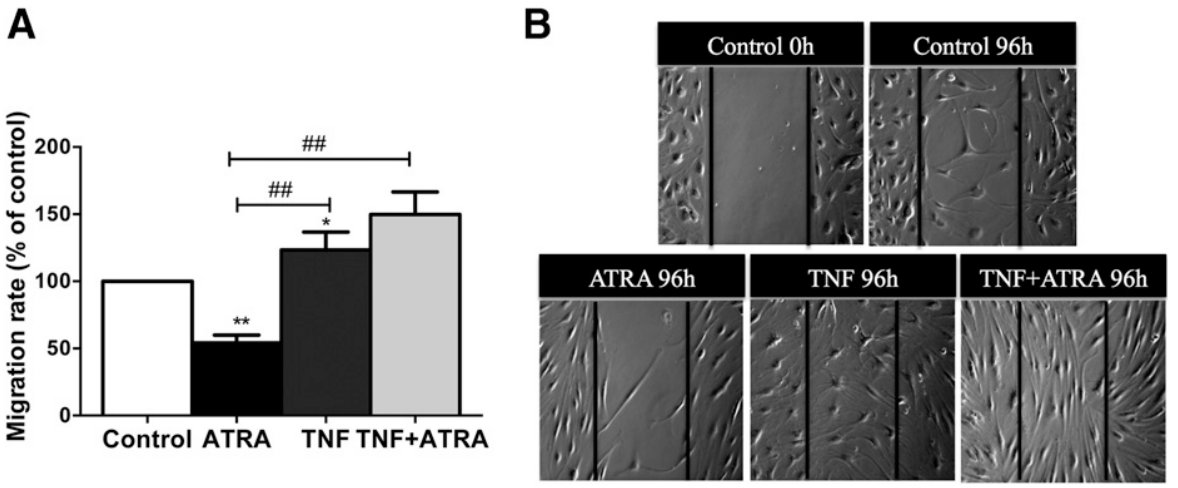

Fig. 1. ATRA reduces RA FLS migration. (A) Migration rate of RA FLS measured by woundhealing assays. RA FLS were stimulated for 96 hours with $5 \mu \mathrm{M}$ ATRA, $10 \mathrm{ng} / \mathrm{ml} \mathrm{TNF}$, or TNF+ATRA. Migration rate reached by RA FLS control was considered as $100 \%$. (B) Representative microphotographs are shown. Values are the mean \pm Standard Error of the Mean (SEM) of FLS from nine RA patients. ${ }^{*} P<0.05$; $* * P<0.01$ vs. control; \#\#P<0.01 between the signaled conditions, by Wilcoxon matchedpairs test. most down-regulated extracellular matrix genes by ATRA were collagen (COL) 5A3, lumican, elastin, tenascin $\mathrm{C}$, COL6A3, COL8A2, COL1A2, COL1A1, and other collagen genes. Six other gene sets showed an enhancement of expression with the combined treatment (Fig. 3). The clearest pattern in this category corresponded to oxidative phosphorylation, which was up-regulated in all ATRA+TNF comparisons without experiencing a significant increase in the wells cultured separately with TNF or with ATRA. A similar pattern was found for the mitotic spindle, DNA repair, adipogenesis, mechanistic target of rapamycin complex 1 signaling, and cholesterol homeostasis gene sets (Fig. 3).

There were four other gene sets showing up-regulated expression associated with ATRA. For three of them, the increase was uniform in all comparisons, indicating that they were stimulated by both ATRA and TNF and further increased by the combination of ATRA+TNF. These three sets are denominated as such: E2F targets, G2M checkpoint, and MYC targets v1. The other set that showed a similar, though less consistent, pattern was $\mathrm{TNF}-\alpha$ signaling via Nuclear factor kappa light chain enhancer of activated B cells (NFkB) (Fig. 3).

The remaining gene sets showing significant enrichment were exclusively related to induction by $\mathrm{TNF}$ and, therefore, of less interest in the current study. However, it is worth noting to signal their substantial number, 12 , and that 11 of them were enriched in up-regulated genes. This bias toward increased gene transcription and the identity of the enhanced gene sets reflected the known effect of TNF in the immune response (Fig. 3).
For the confirmatory experiments, we selected the genes from the first decile of the most significantly enriched pathways (inflammatory response, interferon gamma response, TNF- $\alpha$ signaling via NFKB, and epithelial-mesenchymal transition) that were of interest in cell migration, invasion, motility, or inflammation (Table 2). Some of the 11 genes fulfilling this selection appeared in several pathways, and they were validated by real-time PCR.

Analysis of ATRA and TNF in the Regulation of Specific Genes. Real-time PCR of the 11 selected genes was done with FLS from five to seven patients with RA. The commonest effect of ATRA was to down-regulate gene expression. The decrease was significant for epithelial stromal interaction 1 (EPSTI1), matrix metalloprotease 1 (MMP1), $C$-X3-C motif chemokine ligand 1 (CX3CL1), pentraxin 3 (PTX3), and vascular cell adhesion protein 1 (VCAM1) in the comparison between ATRA and control (Fig. 4). There was also a significant decrease in FLS treated with ATRA+TNF relative to the FLS incubated with TNF (Fig. 4). This reduction was observed for some of the same genes that were downmodulated by ATRA alone, specifically for EPTSI1, $M M P 1$, and VCAM1 (Fig. 4). However, it was also observed that $C-X-C$ motif chemokine ligand 9 (CXCL9), $C-X-C$ motif chemokine ligand 3 (CXCL3), and ST3 beta-galactoside alpha2, 3-sialyltransferase 5 (ST3GAL5) did not demonstrate differences in the ATRA versus control comparison and that nidogen 2 (NID2) showed up-regulation with ATRA alone. However, ATRA only reverted the TNF-induced expression to the control level for MMP1 (Fig. 4). The inability of ATRA to revert TNF effects was equally apparent in the two genes that were down-regulated by TNF, NID2, and ST3GAL5. These
A

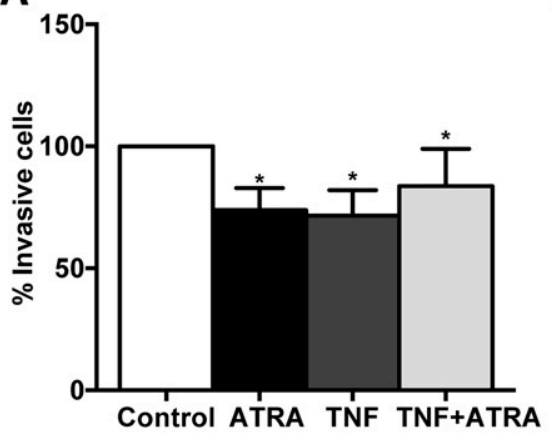

B

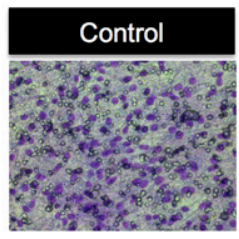

TNF

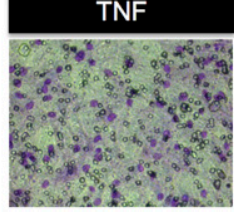

ATRA

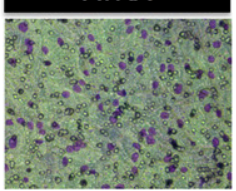

TNF+ATRA

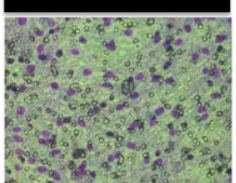

Fig. 2. ATRA reduces RA FLS invasion. (A) Percentage of RA FLS stimulated with or without $5 \mu \mathrm{M}$ ATRA that invaded the inserts coated with Matrigel. Number of cells in untreated controls that invaded was used as $100 \%$. (B) Representative microphotographs are shown. Values are the mean \pm Standard Error of the Mean (SEM) of FLS from nine patients with RA. $* P<0.05$, by Wilcoxon matched-pairs test. 


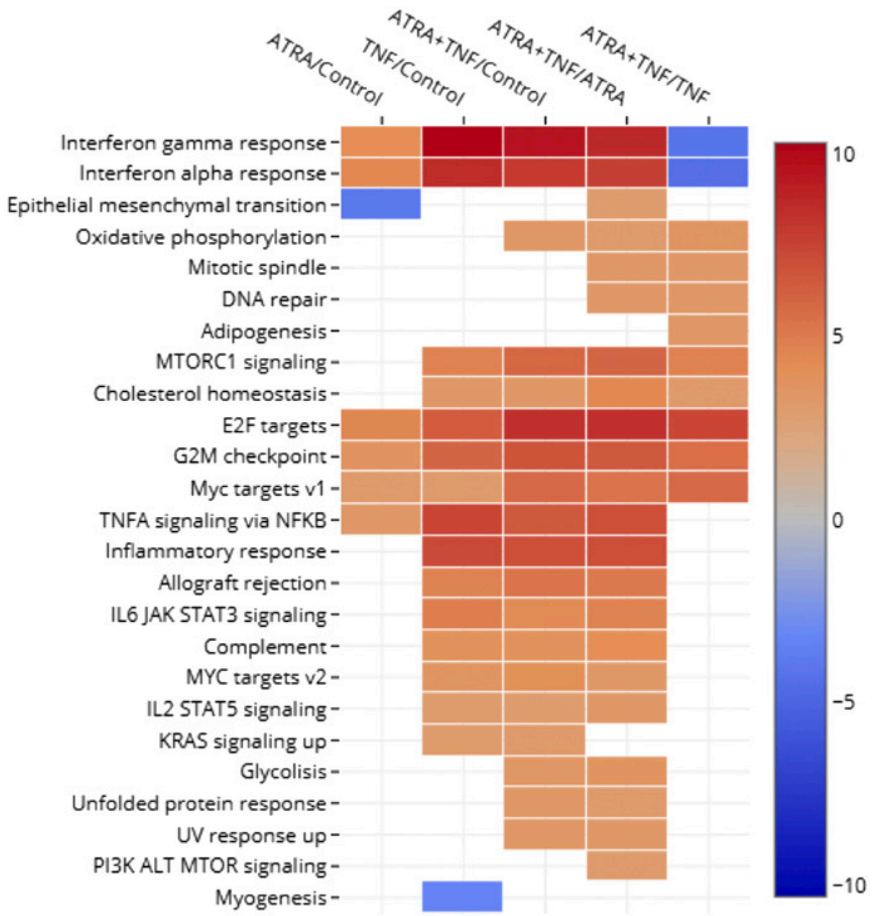

Fig. 3. Heatmap showing the gene sets that were significantly enriched in overexpressed (red) or down-regulated (blue) genes. The scale shows NESs. Columns represent the comparisons of condition 1 /condition 2 . Rows show the gene set names. Significantly enriched gene sets were identified with NES $\geq 3$ and false discovery rate $<10^{-4}$ in GSEA preranked analysis. JAK, Janus kinase; MTOR, mechanistic target of rapamycin; MTORC1, MTOR complex 1; STAT5, signal transducer and activator of transcription 5.

two genes were further down-regulated by the ATRA+TNF treatment in comparison with the treatment with TNF alone. This potentiated down-regulation was particularly striking for NID2 because the NID2 expression was induced by ATRA in the absence of TNF (Fig. 4).

The two remaining genes, nucleotide-binding oligomerization domain 2 (NOD2) and interleukin $1 B$ (IL1B) were not down-regulated by ATRA in any experimental setting but for different reasons. NOD2 expression showed a complete insensitivity to ATRA (Fig. 4). In contrast, expression of IL1B was induced by ATRA, both alone and in the TNF+ATRA combination (Fig. 4).

Dose-Effect Curve of ATRA on the TNF-Induced Migration of RA FLS. To analyze how the interplay between ATRA and TNF affects RA FLS migration at different relative concentrations, we varied the doses of ATRA while keeping the dose of TNF constant (Fig. 5). These experiments showed that ATRA in isolation was able to decrease RA FLS migration even at the lower dose assayed, which was onetenth of that previously used, with further decrease at higher doses. In contrast, the increase in migration observed in the presence of TNF was not significantly modified with the addition of ATRA in the $0.5-10 \mu \mathrm{M}$ range. However, higher doses of ATRA were able to significantly overcome the TNF potentiation ( 0.04 and 0.03 with 20 and $40 \mu \mathrm{M}$, respectively). Specifically, the TNF-induced migration of RA FLS was reduced by $35.6 \%$ with $40 \mu \mathrm{M}$ ATRA (Fig. 5).

\section{Discussion}

Our experiments have identified new areas in which ATRA could control the FLS-activated phenotype. They include a decrease in FLS migration and invasion, which, when uncontrolled, are pivotal contributors to cartilage and bone damage of the inflamed joints. In addition, ATRA showed down-regulatory effect on the expression of specific genes related to cell motility. However, it did not oppose all aspects of the FLS-activated pattern of expression. This complexity of actions was compounded by the strong effect of TNF, which showed a dominant effect on many of the aspects of the RA FLS phenotype. These results integrate appropriately with the growing evidence indicating the contextual dependency of retinoid actions. Interestingly, we also observed that TNF has opposed effects on the migration and invasion of RA FLS. The cause of this discrepancy is unknown. However, it is necessary to note that concordant increased cell migration and invasion is characteristic of metastatic cancer cells not of tissue growth, inflammation, or wound repair, in which cell motility is increased without invasiveness (Wu and Zhou, 2010; Hulkower and Herber, 2011). In RA animal models, synovial invasion and attachment to cartilage and bone and even transmigration to other joints are present, but TNF is not able on its own to promote them. The experiments in Human Tumor Necrosis Factor (hTNF) transgenic mice show that these processes are dependent on the presence of IL-1 or cartilage damage (Korb-Pap et al., 2012; Hillen et al., 2017; Ospelt, 2017).

No previous study has analyzed the role of ATRA, or any other retinoid, in the RA FLS except for our previous work that was restricted to FLS proliferation and survival. FLSs' proliferation was insensitive to ATRA, whereas their resistance to apoptosis was exacerbated (Mosquera et al., 2018). The inhibitory effects of ATRA on FLS migration and invasion observed in the current study were in concordance with that found in cancerous and nonmalignant cells.

TABLE 2

Gene selected to validate microarray analysis

\begin{tabular}{llll}
\hline Gene Set & & & \\
\hline Epithelial-mesenchymal transition & MMP1 & PTX3 & VCAM1 NOD2 \\
Inflammatory response & CX3CL1 & CXCL9 & NOD2 \\
Interferon gamma response & EPSTI1 & VCAM1 & CXCL9 \\
TNF- $\alpha$ signaling via NFKB & PTX3 & CXCL3 & IL1B \\
IL6 JAK STAT3 signaling & CXCL3 & CXCL9 & IL1B \\
Allograft rejection & CXCL9 & IL1B & \\
Interferon alpha response & EPSTI1 & & \\
KRAS signaling up & IL1B & & \\
\hline
\end{tabular}




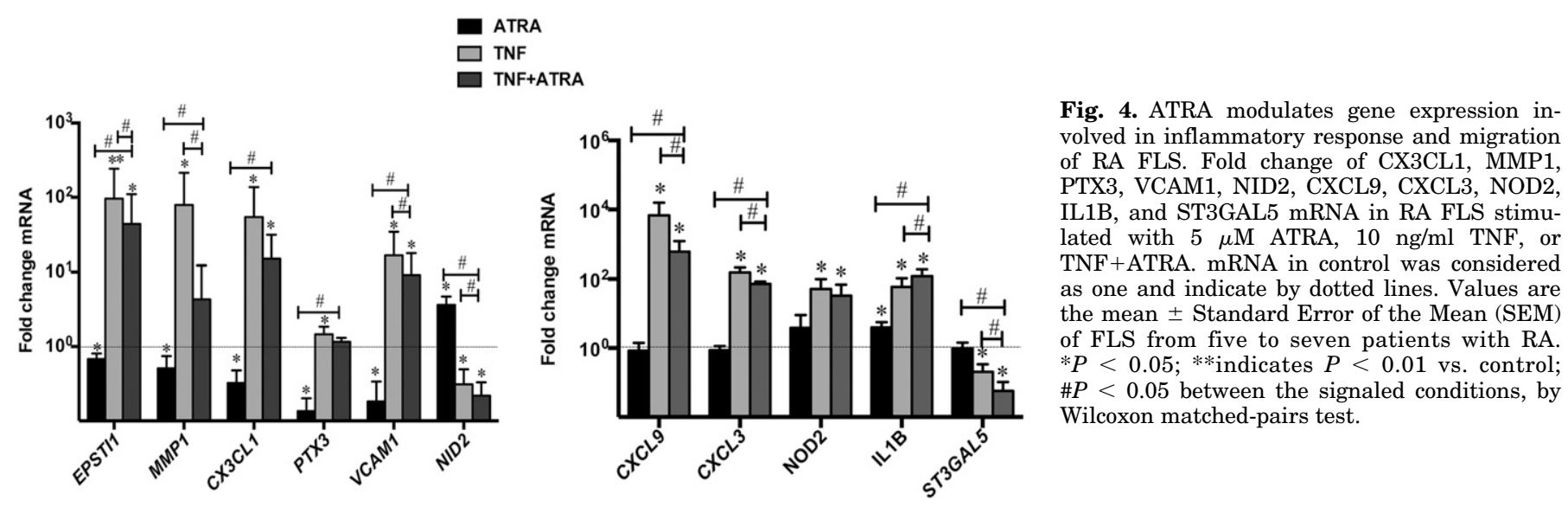

The reduced migration and invasion of cancer cell lines induced by retinoids correlate in animal models with decreased tumor growth and metastases (Tabata et al., 2009; Zhao et al., 2009; Benelli et al., 2010; Applegate and Lane, 2015; Waters et al., 2015; Williams et al., 2018). These effects together with other beneficial actions have motivated the use of retinoids for the treatment of several malignancies, including acute promyelocytic leukemia (Cicconi et al., 2018; Lengfelder et al., 2018), head and neck squamous cell carcinoma (Bhatia et al., 2017), and neuroblastoma (Cheung, 2015).

Gene-set analysis of the microarray data identified a single ATRA down-regulated gene set: the epithelial-mesenchymal transition, which is critical in the RA FLS phenotype (Steenvoorden et al., 2006; Ekwall et al., 2011; Li et al., 2013; Choi et al., 2017). In effect, the FLSs from healthy subjects show some typical markers and functional features of epithelial cells in spite of their mesenchymal origin. These epithelial features are lost more or less completely in the early phases of $\mathrm{RA}$ in a process that resembles the epithelial-mesenchymal transition and that includes enhanced migration and active invasiveness. In addition, the epithelial-mesenchymal transition pathway has been identified as the most up-regulated pathway associated with RA FLS invasiveness in a previous

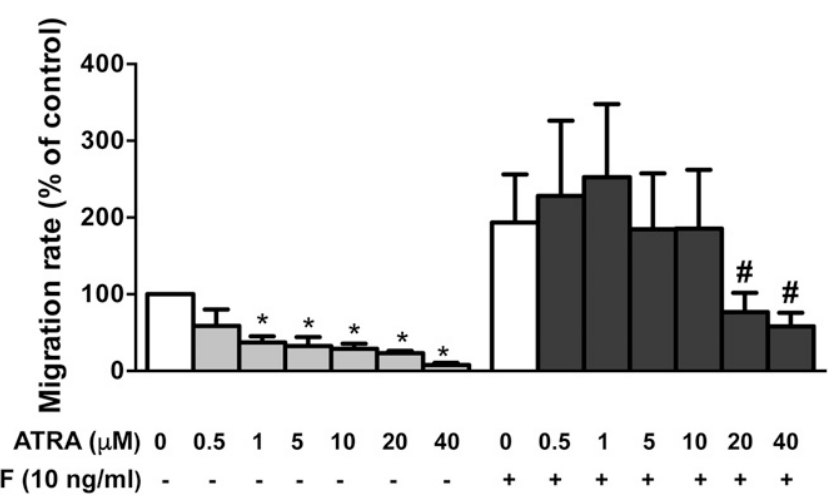

Fig. 5. The interplay between ATRA and TNF on RA FLS migration is dose-dependent. Migration rate of RA FLS measured by wound-healing assays. RA FLSs were stimulated for 96 hours with the indicated ATRA concentrations in presence or absence of $10 \mathrm{ng} / \mathrm{ml} \mathrm{TNF}$. The migration rate reached by control RA FLS was considered as $100 \%$. Values are the mean \pm ESM of FLS from five patients with RA. $* P<0.05$ vs. control without TNF; $\# P<0.05$ vs. control with TNF. transcriptomic study (You et al., 2014). Therefore, downregulation of the epithelial-mesenchymal transition by ATRA could explain the inhibition of migration and invasion we have observed.

Additional genes that could be involved in migration inhibition were suggested by quantitative PCR results. They showed a marked down-regulation with ATRA of CX3CL1 and EPSTI1 together with decreases of MMP1, PTX3, and VCAM1, which are included in the epithelial-mesenchymal transition gene set. All these genes are involved in cell migration and invasion ( $\mathrm{Li}$ et al., 2014; Tan et al., 2016; Tung et al., 2016; Bernardini et al., 2017; Wang et al., 2017; Kong et al., 2018). In effect, VCAM1 participates in the transendothelial migration of leukocytes (Kong et al., 2018), whereas EPST11 (Li et al., 2014; Tan et al., 2016) and PTX3 (Tung et al., 2016; Chan et al., 2017) contribute to cancer invasion and metastasis. Even more relevant is downregulation of CX3CL1 and MMP1, because they participate in RA FLS migration. On one side, CX3CL1 is a chemokine ligand that induces FLS proliferation, migration, and pannus formation in an autocrine way (Bernardini et al., 2017), whereas MMP1 contributes to migration and invasion as an extracellular matrix protease (Huang et al., 2017; Wang et al., 2017).

Another relevant aspect of our results is the modulation of ATRA actions by the presence of TNF. Its most notable manifestation was the reversal of its inhibition of RA FLS migration. The nature of this modulation is not known, but it was possible to reverse it with larger concentrations of ATRA. In addition, $5 \mu \mathrm{M}$ ATRA in combination with TNF modulated the gene expression differently than ATRA alone. Many interaction types were observed in our analysis, from potentiation of the TNF effect to its reversal. Then, in further studies it would be interesting to more thoroughly analyze the effect of TNF+ATRA in these pathways.

The interaction of ATRA and TNF has been previously observed in chondrocytes (Huang et al., 2017) and the Thelper subset differentiation (Basu et al., 2015; Brown and Noelle, 2015). However, no previous evidence of interaction was available in RA or healthy FLSs or in any other fibroblasts. In addition, epidemiologic studies have found a paradoxical disparity attributed to the contextual dependency of the retinoid effect. So, high plasma levels of retinoids are associated with decreased frequency of some chronic diseases (Rubin et al., 2017; Toti et al., 2018). In RA, low levels of retinoids in 
plasma are associated with increased prevalence (Comstock et al., 1997), whereas treatment with retinoids of arthritis models has resulted in a variety of results, from an increase in disease severity (Trentham and Brinckerhoff, 1982; Beehler et al., 2003) to its attenuation (Kuwabara et al., 1996; Kwok et al., 2012).

The beneficial effect of ATRA was observed at the supraphysiological dose of $5 \mu \mathrm{M}$, which has been frequently used in in vitro studies (Chen and Stallings, 2007; Das et al., 2010, 2013; Foley et al., 2011; García-Regalado et al., 2013; Mosquera et al, 2018) and broadly corresponds to the ATRA concentrations attainable with the doses used to markedly supress collagen-induced arthritis in DBA mice (Kwok et al., 2012). In effect, the $0.5 \mathrm{mg} / \mathrm{kg}$ of ATRA given to these mice leads to peak ATRA concentrations between 0.6 and $3 \mu \mathrm{M}$ in serum and tissues, as estimated from the available data in mice (Jing et al., 2017). These concentrations are about 1000 times larger than the endogenous concentration found in the plasma of human subjects (Jing et al., 2017), but there is no reason to expect they will be associated with intolerable adverse effects because they are about one-hundredth the concentrations found in plasma with the recommended dose for the treatment of acute promyelocytic leukemia (Castaigne et al., 1993; Sanz et al., 2009; Osman et al., 2018). Therefore, the $5 \mu \mathrm{M}$ concentration was clearly larger than the endogenous ATRA, sufficient for eliciting significant effects in preclinical arthritis models, and likely to be well tolerated.

The dominance of TNF over ATRA on RA FLS reinforces the need for complementary approaches when considering drugs aimed to control the RA FLS phenotype. This idea is widely accepted (Niedermeier et al., 2010). The experience of drugs targeting synoviocytes in RA does not go beyond preclinical studies (Dong-Liang et al., 2016). However, several therapies intended to reduce synovial hyperplasia, including radioactive, surgical, and arthroscopic synovectomy, are still applied with success in refractory patients with RA (Chalmers et al., 2011; Lee et al., 2014; Knut, 2015).

The possibility that ATRA could be used for the treatment of RA has been pursued only in preclinical studies, as already mentioned (Kuwabara et al., 1996; Kwok et al., 2012). However, the clinical experience of combining treatments in $\mathrm{RA}$ is extensive. Current recommendations include the combination of a nontargeted drug, such as methotrexate, with a targeted drug, such as an anti-TNF monoclonal antibody, to treat the patients that show inadequate response (Smolen et al., 2017). The significant fraction of patients that still do not respond has motivated the search for other combinations. Therefore, a combination of drugs targeting inflammation and the synoviocytes will follow in this tradition.

Overall, the data shown here have demonstrated for the first time that ATRA can modulate the aggressive phenotype of FLS from patients with RA. On practical grounds, it seems reasonable to consider that combined treatments will include drugs to control inflammation and autoimmunity in addition to the RA FLS-directed drug. Therefore, future preclinical trials should consider the many effects of retinoids, including the effects on FLS, the interactions with TNF, and other contextual influences. It is possible that in this way, the effect of retinoids on arthritis models could be clarified and the path toward clinical trials could be open in the context of drug repositioning.

\section{Acknowledgments}

We thank Carmen Pena for excellent technical assistance and the patients for their contribution. The authors also thank for technical and human support provided by Servicios Generales de Investigación (SGIker) of Universidad del País Vasco/Euskal Herriko Unibertsitatea (UPV/EHU) and European funding [European Regional Development Fund (ERDF) and European Social Fund (ESF)].

\section{Authorship Contributions}

Participation in research design: Gonzalez, Conde.

Conducted experiments: Mosquera, Rodriguez-Trillo.

Performed data analysis: Mosquera, Rodriguez-Trillo, Blanco, Mera-Varela, Gonzalez, Conde.

Wrote or contributed to writing of the manuscript: Mosquera, Rodriguez-Trillo, Blanco, Mera-Varela, Gonzalez, Conde.

\section{References}

Al Tanoury Z, Piskunov A, and Rochette-Egly C (2013) Vitamin A and retinoid signaling: genomic and nongenomic effects. J Lipid Res 54:1761-1775.

Applegate CC and Lane MA (2015) Role of retinoids in the prevention and treatment of colorectal cancer. World J Gastrointest Oncol 7:184-203.

Arnett FC, Edworthy SM, Bloch DA, McShane DJ, Fries JF, Cooper NS, Healey LA, Kaplan SR, Liang MH, Luthra HS, et al. (1988) The American Rheumatism Association 1987 revised criteria for the classification of rheumatoid arthritis. Arthritis Rheum 31:315-324.

Bartok B and Firestein GS (2010) Fibroblast-like synoviocytes: key effector cells in rheumatoid arthritis. Immunol Rev 233:233-255.

Basu R, Whitley SK, Bhaumik S, Zindl CL, Schoeb TR, Benveniste EN, Pear WS, Hatton RD, and Weaver CT (2015) IL-1 signaling modulates activation of STAT transcription factors to antagonize retinoic acid signaling and control the TH17 cell-iTreg cell balance. Nat Immunol 16:286-295.

Beehler BC, Hei YJ, Chen S, Lupisella JA, Ostrowski J, Starrett JE, Tortolani D, Tramposch KM, and Reczek PR (2003) Inhibition of disease progression by a novel retinoid antagonist in animal models of arthritis. J Rheumatol 30:355-363.

Benelli R, Monteghirfo S, Venè R, Tosetti F, and Ferrari N (2010) The chemopreventive retinoid $4 \mathrm{HPR}$ impairs prostate cancer cell migration and invasion by interfering with FAK/AKT/GSK3beta pathway and beta-catenin stability. Mol Cancer 9:142.

Bernardini G, Benigni G, Scrivo R, Valesini G, and Santoni A (2017) The multifunctional role of the chemokine system in arthritogenic processes. Curr Rheumatol Rep 19:11.

Bhatia AK, Lee JW, Pinto HA, Jacobs CD, Limburg PJ, Rubin P, Arusell RM, Dunphy EP, Khandekar JD, Reiner SA, et al. (2017) Double-blind, randomized phase 3 trial of low-dose 13-cis retinoic acid in the prevention of second primaries in head and neck cancer: long-term follow-up of a trial of the Eastern Cooperative Oncology Group-ACRIN Cancer Research Group (C0590). Cancer 123:4653-4662.

Brown CC and Noelle RJ (2015) Seeing through the dark: new insights into the immune regulatory functions of vitamin A. Eur J Immunol 45:1287-1295.

Castaigne S, Lefebvre P, Chomienne C, Suc E, Rigal-Huguet F, Gardin C, Delmer A Archimbaud E, Tilly H, Janvier M, et al. (1993) Effectiveness and pharmacokinetics of low-dose all-trans retinoic acid $(25 \mathrm{mg} / \mathrm{m} 2)$ in acute promyelocytic leukemia. Blood 82:3560-3563.

Chalmers PN, Sherman SL, Raphael BS, and Su EP (2011) Rheumatoid synovectomy: does the surgical approach matter? Clin Orthop Relat Res 469:2062-2071.

Chan SH, Tsai JP, Shen CJ, Liao YH, and Chen BK (2017) Oleate-induced PTX3 promotes head and neck squamous cell carcinoma metastasis through the upregulation of vimentin. Oncotarget 8:41364-41378.

Chen Y and Stallings RL (2007) Differential patterns of microRNA expression in neuroblastoma are correlated with prognosis, differentiation, and apoptosis. Cancer Res 67:976-983.

Cheung BB (2015) Combination therapies improve the anticancer activities of retinoids in neuroblastoma. World J Clin Oncol 6:212-215.

Choi IY, Karpus ON, Turner JD, Hardie D, Marshall JL, de Hair MJH, Maijer KI, Tak PP, Raza K, Hamann J, et al. (2017) Stromal cell markers are differentially expressed in the synovial tissue of patients with early arthritis. PLoS One 12: e0182751.

Cicconi L, Breccia M, Franceschini L, Latagliata R, Molica M, Divona M, Diverio D, Rizzo M, Ottone T, Iaccarino L, et al. (2018) Prolonged treatment with arsenic trioxide (ATO) and all-trans-retinoic acid (ATRA) for relapsed acute promyelocytic leukemia previously treated with ATRA and chemotherapy. Ann Hematol 97: 1797-1802.

Comstock GW, Burke AE, Hoffman SC, Helzlsouer KJ, Bendich A, Masi AT, Norkus EP, Malamet RL, and Gershwin ME (1997) Serum concentrations of alpha tocopherol, beta carotene, and retinol preceding the diagnosis of rheumatoid arthritis and systemic lupus erythematosus. Ann Rheum Dis 56:323-325.

Coyle KM, Sultan M, Thomas ML, and Vaghar-Kashani A (2013) Retinoid signaling in cancer and its promise for therapy. J Carcinog Mutagen S7:1-14.

Cui J, Gong M, He Y, Li Q, He T, and Bi Y (2016) All-trans retinoic acid inhibits proliferation, migration, invasion and induces differentiation of hepa1-6 cells through reversing EMT in vitro. Int $J$ Oncol 48:349-357.

Das S, Bryan K, Buckley PG, Piskareva O, Bray IM, Foley N, Ryan J, Lynch J, Creevey L, Fay J, et al. (2013) Modulation of neuroblastoma disease pathogenesis by an extensive network of epigenetically regulated microRNAs. Oncogene 32: 2927-2936. 
Das S, Foley N, Bryan K, Watters KM, Bray I, Murphy DM, Buckley PG, and Stallings RL (2010) MicroRNA mediates DNA demethylation events triggered by retinoic acid during neuroblastoma cell differentiation. Cancer Res 70: $7874-7881$.

Ekwall AK, Eisler T, Anderberg C, Jin C, Karlsson N, Brisslert M, and Bokarewa MI (2011) The tumour-associated glycoprotein podoplanin is expressed in fibroblastlike synoviocytes of the hyperplastic synovial lining layer in rheumatoid arthritis. Arthritis Res Ther 13:R40.

Flamini MI, Gauna GV, Sottile ML, Nadin BS, Sanchez AM, and Vargas-Roig LM (2014) Retinoic acid reduces migration of human breast cancer cells: role of retinoic acid receptor beta. J Cell Mol Med 18:1113-1123.

Foley NH, Bray I, Watters KM, Das S, Bryan K, Bernas T, Prehn JH, and Stallings RL (2011) MicroRNAs 10a and 10b are potent inducers of neuroblastoma cell differentiation through targeting of nuclear receptor corepressor 2. Cell Death Differ 18:1089-1098.

García-Regalado A, Vargas M, García-Carrancá A, Aréchaga-Ocampo E, and González-De la Rosa CH (2013) Activation of Akt pathway by transcriptionindependent mechanisms of retinoic acid promotes survival and invasion in lung cancer cells. Mol Cancer 12:44.

Hillen J, Geyer C, Heitzmann M, Beckmann D, Krause A, Winkler I, Pavenstädt H Bremer C, Pap T, and Korb-Pap A (2017) Structural cartilage damage attracts circulating rheumatoid arthritis synovial fibroblasts into affected joints. Arthritis Res Ther 19:40.

Hulkower KI and Herber RL (2011) Cell migration and invasion assays as tools for drug discovery. Pharmaceutics 3:107-124.

Jing J, Nelson C, Paik J, Shirasaka Y, Amory JK, and Isoherranen N (2017) Physiologically based pharmacokinetic model of all-trans-retinoic acid with application to cancer populations and drug interactions. $J$ Pharmacol Exp Ther 361:246-258.

Klareskog L, Catrina AI, and Paget S (2009) Rheumatoid arthritis. Lancet 373 $659-672$

Knut L (2015) Radiosynovectomy in the therapeutic management of arthritis. World J Nucl Med 14:10-15.

Kong DH, Kim YK, Kim MR, Jang JH, and Lee S (2018) Emerging roles of vascular cell adhesión molecule-1 (VCAM-1) in immunological disorders and cancer. Int J Mol Sci 19:44.

Korb-Pap A, Stratis A, Mühlenberg K, Niederreiter B, Hayer S, Echtermeyer F, Stange R, Zwerina J, Pap T, Pavenstädt H, et al. (2012) Early structural changes in cartilage and bone are required for the attachment and invasion of inflamed synovial tissue during destructive inflammatory arthritis. Ann Rheum Dis 71: 1004-1011.

Kuwabara K, Shudo K, and Hori Y (1996) Novel synthetic retinoic acid inhibits rat collagen arthritis and differentially affects serum immunoglobulin subclass levels. FEBS Lett 378:153-156.

Kwok SK, Park MK, Cho ML, Oh HJ, Park EM, Lee DG, Lee J, Kim HY, and Park SH (2012) Retinoic acid attenuates rheumatoid inflammation in mice. J Immunol 189 1062-1071.

Lee HI, Lee KH, Koh KH, and Park MJ (2014) Long-term results of arthroscopic wrist synovectomy in rheumatoid arthritis. J Hand Surg Am 39:1295-1300.

Lengfelder E, Görlich D, Nowak D, Spiekermann K, Haferlach C, Krug U, Kreuzer KA, Braess J, Schliemann C, Lindemann HW, et al.; German Acute Myeloid Leukemia Cooperative Group (AMLCG) (2018) Frontline therapy of acute promyelocytic leukemia: randomized comparison of ATRA and intensified chemotherapy versus ATRA and anthracyclines. Eur J Haematol 100:154-162.

Li N, Lu Y, Li D, Zheng X, Lian J, Li S, Cui H, Zhang L, Sang L, Wang Y, et al. (2017) All-trans retinoic acid suppresses the angiopoietin-Tie2 pathway and inhibits angiogenesis and metastasis in esophageal squamous cell carcinoma. PLoS One 12 e0174555.

Li GQ, Zhang Y, Liu D, Qian YY, Zhang H, Guo SY, Sunagawa M, Hisamitsu T, and Liu YQ (2013) PI3 kinase/Akt/HIF-1 $\alpha$ pathway is associated with hypoxiainduced epithelial-mesenchymal transition in fibroblast-like synoviocytes of rheumatoid arthritis. Mol Cell Biochem 372:221-231.

Li T, Lu H, Shen C, Lahiri SK, Wason MS, Mukherjee D, Yu L, and Zhao J (2014) Identification of epithelial stromal interaction 1 as a novel effector downstream of Krüppel-like factor 8 in breast cancer invasion and metastasis. Oncogene 33: $4746-4755$.

Lories RJ, Derese I, De Bari C, and Luyten FP (2003) In vitro growth rate of fibroblast-like synovial cells is reduced by methotrexate treatment. Ann Rheum Dis 62:568-571.

Massacesi L, Castigli E, Vergelli M, Olivotto J, Abbamondi AL, Sarlo F, and Amaducci L (1991) Immunosuppressive activity of 13-cis-retinoic acid and prevention of experimental autoimmune encephalomyelitis in rats. J Clin Invest 88:1331-1337.

McInnes IB and Schett G (2011) The pathogenesis of rheumatoid arthritis. $N$ Engl $J$ Med 365:2205-2219.

Mosquera N, Rodriguez-Trillo A, Mera-Varela A, Gonzalez A, and Conde C (2018) Uncovering cellular retinoic acid-binding protein 2 as a potential target for rheumatoid arthritis synovial hyperplasia. Sci Rep 8:8731.

Neumann E, Lefêvre S, Zimmermann B, Gay S, and Müller-Ladner U (2010) Rheumatoid arthritis progression mediated by activated synovial fibroblasts. Trends Mol Med 16:458-468.
Niedermeier M, Pap T, and Korb A (2010) Therapeutic opportunities in fibroblasts in inflammatory arthritis. Best Pract Res Clin Rheumatol 24:527-540.

Orosa B, Gonzalez A, Mera A, Gomez-Reino JJ, and Conde C (2012) Lysophosphatidic acid receptor 1 suppression sensitizes rheumatoid fibroblast-like synoviocytes to TNF-induced apoptosis. Arthritis Rheum 64:2460-2470.

Osman AEG, Anderson J, Churpek JE, Christ TN, Curran E, Godley LA, Liu H, Thirman MJ, Odenike T, Stock W, et al. (2018) Treatment of acute promyelocytic leukemia in adults. $J$ Oncol Pract 14:649-657.

Ospelt C (2017) Synovial fibroblasts in 2017. RMD Open 3:e000471.

Pérez de Lema G, Lucio-Cazaña FJ, Molina A, Luckow B, Schmid H, de Wit C, Moreno-Manzano V, Banas B, Mampaso F, and Schlöndorff D (2004) Retinoic acid treatment protects MRL/lpr lupus mice from the development of glomerular disease. Kidney Int 66:1018-1028.

Rosengren S, Boyle DL, and Firestein GS (2007) Acquisition, culture, and phenotyping of synovial fibroblasts. Methods Mol Med 135:365-375.

Rubin LP, Ross AC, Stephensen CB, Bohn T, and Tanumihardjo SA (2017) Metabolic effects of inflammation on vitamin $\mathrm{A}$ and carotenoids in humans and animal models. Adv Nutr 8:197-212.

Sanz MA, Grimwade D, Tallman MS, Lowenberg B, Fenaux P, Estey EH, Naoe T, Lengfelder E, Büchner T, Döhner H, et al. (2009) Management of acute promyelocytic leukemia: recommendations from an expert panel on behalf of the European LeukemiaNet. Blood 113:1875-1891.

Smolen JS, Landewé R, Bijlsma J, Burmester G, Chatzidionysiou K, Dougados M, Nam J, Ramiro S, Voshaar M, van Vollenhoven R, et al. (2017) EULAR recommendations for the management of rheumatoid arthritis with synthetic and biological disease-modifying antirheumatic drugs: 2016 update. Ann Rheum Dis 76: 960-977.

Steenvoorden MM, Tolboom TC, van der Pluijm G, Löwik C, Visser CP, DeGroot J, Gittenberger-DeGroot AC, DeRuiter MC, Wisse BJ, Huizinga TW, et al. (2006) Transition of healthy to diseased synovial tissue in rheumatoid arthritis is associated with gain of mesenchymal/fibrotic characteristics. Arthritis Res Ther 8:R165. Tabata C, Tabata R, Hirayama N, Yasumitsu A, Yamada S, Murakami A, Iida S, Tamura K, Terada T, Kuribayashi K, et al. (2009) All-trans-retinoic acid inhibits tumour growth of malignant pleural mesothelioma in mice. Eur Respir $J$ 34: 1159-1167.

Tan YY, Xu XY, Wang JF, Zhang CW, and Zhang SC (2016) MiR-654-5p attenuates breast cancer progression by targeting EPSTI1. Am J Cancer Res 6:522-532.

Toti E, Chen CO, Palmery M, Villaño Valencia D, and Peluso I (2018) Nonprovitamin A and Provitamin A carotenoids as immunomodulators: recommended dietary allowance, therapeutic index, or personalized nutrition? Oxid Med Cell Longev 2018:4637861.

Trentham DE and Brinckerhoff CE (1982) Augmentation of collagen arthritis by synthetic analogues of retinoic acid. $J$ Immunol 129:2668-2672.

Tung JN, Ko CP, Yang SF, Cheng CW, Chen PN, Chang CY, Lin CL, Yang TF, Hsieh $\mathrm{YH}$, and Chen KC (2016) Inhibition of pentraxin 3 in glioma cells impairs proliferation and invasion in vitro and in vivo. $J$ Neurooncol 129:201-209.

Wang Y, Zhong YJ, Wang YY, Xing J, and Wang ZM (2016) All-trans retinoic acid prevents the development of type 1 diabetes by affecting the levels of interferon gamma and interleukin 4 in streptozotocin-induced murine diabetes model. Genet Mol Res 15:1-9.

Wang W, Liu J, Yang B, Ma Z, Liu G, Shen W, and Zhang Y (2017) Modulation of platelet-derived microparticles to adhesion and motility of human rheumatoid arthritis fibroblast-like synoviocytes. PLoS One 12:e181003.

Waters AM, Stewart JE, Atigadda VR, Mroczek-Musulman E, Muccio DD, Grubbs CJ, and Beierle EA (2015) Preclinical evaluation of a novel RXR agonist for the treatment of neuroblastoma. Mol Cancer Ther 14:1559-1569.

Williams AP, Waters AM, Stewart JE, Atigadda VR, Mroczek-Musulman E, Muccio DD, Grubbs CJ, and Beierle EA (2018) A novel retinoid X receptor agonist, UAB30, inhibits rhabdomyosarcoma cells in vitro. J Surg Res 228:54-62.

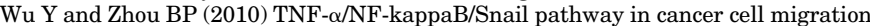
and invasion. $\mathrm{Br} J$ Cancer 102:639-644.

You S, Yoo S-A, Choi S, Kim JY, Park SJ, Ji JD, Kim TH, Kim KJ, Cho CS, Hwang D, et al. (2014) Identification of key regulators for the migration and invasion of rheumatoid synoviocytes through a systems approach. Proc Natl Acad Sci USA 111:550-555.

Young MJ, Wu YH, Chiu WT, Weng TY, Huang YF, and Chou CY (2015) All-trans retinoic acid downregulates ALDH1-mediated stemness and inhibits tumour formation in ovarian cancer cells. Carcinogenesis 36:498-507.

Zhao X, Graves C, Ames SJ, Fisher DE, and Spanjaard RA (2009) Mechanism of regulation and suppression of melanoma invasiveness by novel retinoic acid receptor-gamma target gene carbohydrate sulfotransferase 10. Cancer Res 69: $5218-5225$

Address correspondence to: Carmen Conde, Laboratorio de Reumatología Experimental y Observacional, Instituto de Investigación Sanitaria de Santiago (IDIS), Hospital Clínico Universitario de Santiago de Compostela (CHUS), Servizo Galego de Saude (SERGAS), Travesía da Choupana s/n, Santiago de Compostela 15706, Spain. E-mail: Carmen.Conde.Muro@sergas.es 